\title{
The Pursuit of Excellence in Acute Stroke Care
}

Can. J. Neurol. Sci. 2005; 32: 401-402

Intravenous tPA was approved as treatment for acute ischemic stroke in Canada in February 1999. In this issue of the Canadian Journal of the Neurological Sciences, the Canadian Stroke Network (CSN) report on their findings from phases 1 and 2 of the Registry of the CSN (RCSN). ${ }^{1}$ A phase 3 study is ongoing. The main points of the study are that: 1) a greater percentage of Canadian patients in this cohort receive tissue plasminogen activator (tPA) $(8.9 \%)$ compared with United States patients (typically less than $5 \%^{2}$ ), 2) only $28.3 \%$ of patients are treated within 60 minutes of arrival to the emergency department, and 3) in spite of a protocol violation rate of $27.7 \%$, symptomatic intracranial hemorrhage rates remained low (4.3\%).

The reasons for a higher percentage of patients receiving tPA in Canada are not entirely clear but may be partly related to a greater partnership between hospitals as compared to the United States. Bypass and transfer protocols have facilitated treatment of patients in remote areas who might otherwise not have had access to acute treatment. ${ }^{3,4}$ Though an $8.9 \%$ treatment rate is very good, rates of $15-20 \%$ are achievable..$^{5,6}$ However, achieving such a target requires considerable effort and mobilization of resources into dedicated stroke treatment centers. ${ }^{7}$ The increase in treatment rate from $7.9 \%$ in phase 1 to $10.2 \%$ in phase 2 of this registry suggests that such processes are ongoing.

Aside from patients arriving too late after symptom onset, delay in treatment of patients remains the most significant problem of delivering tPA. Even though $50 \%$ of patients in this study arrived in the emergency department less than 75 minutes after symptom onset, median time to treatment was approximately 84 minutes (including a median time from completion of CT scan to treatment of 46.3 minutes). To put these numbers in perspective, the Heart and Stroke Foundation of Canada, the Canadian Stroke Society, and the American Stroke Association recommend arrival-to-treatment times of under 60 minutes. ${ }^{8,9}$ A number of studies, including this one, clearly show an inverse relationship between time remaining to give tPA and time to treatment from arrival i.e. the less time is left, the faster the treatment time. ${ }^{4,10,11}$ Though there is a natural tendency to wait until a deadline before completing a task, this strategy is detrimental to the acute stroke patient. Earlier treatment is associated with a better outcome, especially when done within 90 minutes of symptom onset. ${ }^{12}$ A number of strategies can be employed to reduce delay including: prioritizing stroke patients for imaging; performing the discussion process with patient and family prior to, or during, imaging; and making thrombolytic materials immediately available prior to, or during, imaging.

The rate of protocol violation in this study $(27.7 \%)$ is comparable to other studies where protocol violation rates range from $30-50 \%,{ }^{2,13}$ and partly reflects the eagerness of physicians to treat patients. The two main protocol violations in this study, which accounted for $91 \%$ of all violations, were systolic blood pressure greater than $185 \mathrm{mmHg}(55.2 \%)$ and symptom onset greater than three hours $(35.8 \%)$. Though the former violation is thought to increase the risk of symptomatic intracranial hemorrhage, there was only one death out of 37 such patients. The values of blood pressures above $185 \mathrm{mmHg}$ are not reported but this finding bears further study. With respect to treatment beyond the three-hour window, a pooled analysis of randomized trials found that therapy up to 4.5 hours might be beneficial, though later treatment times are associated with considerably less efficacy. ${ }^{14}$ Treating physicians in this study may have reasoned that there is little biological difference between a patient who is three hours from symptom onset and another who is three hours and ten minutes from symptom onset. Current guidelines, however, recommend treatment only of those patients who are within the three-hour treatment window. ${ }^{15}$

One other interesting side note to this study is the issue of consent when collecting information on patient treatment. Because of the informed consent requirements, the authors were only able to study $66.1 \%$ of patients treated with tPA in this registry. Patients who did not or could not provide consent had more severe strokes and were more likely to die in hospital. ${ }^{16}$ This selection bias may have led to important errors of detecting effects of treatment and protocol violations. For the phase 3 study, the Ethics Review Boards of the participating institutions have waived consent which will give a more accurate depiction of stroke treatment in Canada.

It is an exciting time in stroke treatment. Once considered a hopeless condition, many physicians are encouraged by positive trial results for both ischemic and hemorrhagic stroke. Findings from the RCSN and the Canadian Activase for Stroke Effectiveness Study ${ }^{17}$ will help further our understanding as to what successes can be achieved and what work lies ahead.

\section{Brian Silver \\ Detroit, Michigan, USA}

\section{REFERENCES}

1. Canadian Stroke Network. Descriptive analysis of tPA use for stroke in phase 1 and 2 of the registry of the Canadian Stroke Network. Can J Neurol Sci 2005;32 (In print)

2. Katzan IL, Furlan AJ, Lloyd LE, et al. Use of tissue-type plasminogen activator for acute ischemic stroke: the Cleveland area experience. JAMA 2000;283:1151-1158. 
3. Riopelle RJ, Howse DC, Bolton C, et al. Regional access to acute ischemic stroke intervention. Stroke 2001;32:652-655.

4. Merino JG, Silver B, Wong E, et al. Extending tissue plasminogen activator use to community and rural stroke patients. Stroke 2002;33:141-146.

5. Grotta JC, Burgin WS, El-Mitwalli A, et al. Intravenous tissue-type plasminogen activator therapy for ischemic stroke: Houston experience 1996 to 2000. Arch Neurol 2001;58:2009-2013.

6. Saposnik G, Young B, Silver B, et al. Lack of improvement in patients with acute stroke after treatment with thrombolytic therapy: predictors and association with outcome. JAMA 2004;292:1839-1844.

7. Alberts MJ, Hademenos G, Latchaw RE, et al. Recommendations for the establishment of primary stroke centers. Brain Attack Coalition. JAMA 2000;283:3102-3109.

8. The Heart and Stroke Foundation of Canada and the Canadian Stroke Society. Stroke Treatment Education Program. 1999; http://209.5.25.171/ClientImages/1/CSSSTEP.pdf (accessed 5-20-05)

9. Adams HP Jr, Brott TG, Furlan AJ, et al. Guidelines for Thrombolytic Therapy for Acute Stroke: a Supplement to the Guidelines for the Management of Patients with Acute Ischemic Stroke. A statement for healthcare professionals from a Special Writing Group of the Stroke Council, American Heart Association. Stroke 1996;27:1711-1718.

10. Chapman KM, Woolfenden AR, Graeb D, et al. Intravenous tissue plasminogen activator for acute ischemic stroke: A Canadian hospital's experience. Stroke 2000;31:2920-2924.

11. Albers GW, Bates VE, Clark WM, et al. Intravenous tissue-type plasminogen activator for treatment of acute stroke: the Standard Treatment with Alteplase to Reverse Stroke (STARS) study. JAMA 2000;283:1145-1150.
12. Marler JR, Tilley BC, Lu M, et al. Early stroke treatment associated with better outcome: the NINDS rt-PA stroke study. Neurology 2000;55:1649-1655.

13. Tanne D, Bates VE, Verro $P$, et al. Initial clinical experience with IV tissue plasminogen activator for acute ischemic stroke: a multicenter survey. The t-PA Stroke Survey Group. Neurology 1999;53:424-427.

14. Hacke W, Donnan G, Fieschi C, et al. Association of outcome with early stroke treatment: pooled analysis of ATLANTIS, ECASS, and NINDS rt-PA stroke trials. Lancet 2004;363:768-774.

15. Adams H, Adams R, Del Zoppo G, Goldstein LB. Guidelines for the early management of patients with ischemic stroke: 2005 guidelines update a scientific statement from the Stroke Council of the American Heart Association/American Stroke Association. Stroke 2005;36:916-923.

16. Tu JV, Willison DJ, Silver FL, et al. Impracticability of informed consent in the Registry of the Canadian Stroke Network. N Engl J Med 2004;350:1414-1421.

17. Hill MD, Buchan AM. Thrombolysis for acute ischemic stroke: results of the Canadian Alteplase for Stroke Effectiveness Study. CMAJ 2005;172:1307-1312. 